\title{
Investigation of Data Quality in a Wireless Sensing Unit Composed of Off-the-Shelf Components
}

\author{
Jin-Song Pei ${ }^{a}$, Chetan Kapoor ${ }^{b}$, Troy L. Graves-Abe ${ }^{c}$, \\ Yohanes P. Sugeng ${ }^{d}$, Nadim Ferzli ${ }^{b}$ and Jerome P. Lynch ${ }^{e}$ \\ ${ }^{a}$ Assistant Professor, School of CEES, University of Oklahoma, Norman, OK 731019, USA \\ ${ }^{b}$ Graduate Student, School of ECE, University of Oklahoma, Norman, OK 731019, USA \\ ${ }^{c}$ PRISM, Department of EE, Princeton University, Princeton, NJ 08544, USA \\ ${ }^{d}$ Graduate Student, School of CEES, University of Oklahoma, Norman, OK 731019, USA \\ ${ }^{e}$ Assistant Professor, Department of CEE, University of Michigan, Ann Arbor, MI 48109, USA
}

\begin{abstract}
This paper presents the preliminary findings of a study on data and system identification results (derived from collected data) in a wireless sensing environment. The goal of this study is to understand how various hardware design choices and operational conditions affect the quality of the data and accuracy of the identified results; the focus of this paper is packet and data loss. A series of experimental investigations are carried out using a laboratory shaking table instrumented with off-the-shelf Micro-Electro-Mechanical Systems (MEMS) accelerometers. A wireless sensing unit is developed to interface with these wired analog accelerometers to enable wireless data transmission. To reduce the overall design variance and aid convenient application in civil infrastructure health monitoring, this wireless unit is built with off-the-shelf microcontroller and radio development boards. The antialiasing filter and analog-to-digital convectors (ADC) are the only customized components in the hardware. By varying critical hardware configurations, including using analog accelerometers of different commercial brands, taking various designs for the anti-aliasing filter, and adopting ADCs with different resolutions, shaking table tests are repeated, the collected data are processed, and the results are compared. Operational conditions such as sampling rate and wireless data transmitting range are also altered separately in the repeated testing. In all of the cases tested, data is also collected using a wire-based data acquisition system to serve as a performance baseline for evaluation of the wireless data transmission performance. Based on this study, the challenges in the hardware design of wireless sensing units and data processing are identified.
\end{abstract}

Keywords: Wireless sensing, packet delivery performance, loopback test, data loss, shaking table test

\section{INTRODUCTION}

Although it has been less than a decade since wireless sensing technology has been applied to structural health monitoring, much literature has recently emerged in this fertile cross-disciplinary research area. Successes have been achieved in the development of academic wireless sensing unit prototypes to which arbitrary analog sensors can be interfaced, wireless data transmission in a decentralized sensor network, ${ }^{1-3}$ and embedded processing performed using digital signal processing (DSP) cores. ${ }^{4}$ In addition to academic efforts, stand-alone wireless sensors and sensor-networks have also been explored by the civil-engineering community. ${ }^{5-7}$

The reliability of the communication signal has been recognized as a critical technical limitation of wireless communications that must be addressed to assure reliable data delivery of sensed data. ${ }^{8}$ The reliability of wireless communications can be reduced by noise, path losses, multi-path effects and physical interference. After years of active research, there exists a large body of literature that has been dedicated to this critical issue. ${ }^{9-13}$ For example, Frequency Hopping Spread Spectrum (FHSS) techniques have been demonstrated as one possible solution. ${ }^{14,15}$

Further author information: (Send correspondence to Jin-Song Pei)

Jin-Song Pei: E-mail: jspei@ou.edu, Telephone: 1405325 4272, Address: School of Civil Engineering and Environmental Science, University of Oklahoma, 202 W. Boyd St., Room 324, Norman, OK 73019-1024, USA 
In structural health monitoring, the reliability of the damage detection methods adopted are an equally critical issue. For example, a noticeable loss of packets using Crossbow MICA motes in the streaming mode compared to traditionally tethered sensors has been cited. ${ }^{16}$ Data losses during the testing of the same wireless sensor platform (Crossbow MICA Mote) has been reported in another study. ${ }^{5}$ Furthermore, others have identified low analog-to-digital conversion (ADC) resolutions as the source of poor low-frequency performance of MEMS accelerometers. ${ }^{6}$

These interesting findings presented in previous studies have motivated this work. Structural health monitoring systems are only possible when the development of hardware and embedded software are integrated. ${ }^{17}$ It can be seen that a systematic study is desirable for better understanding of how various hardware design issues (e.g., the selection of ADC resolution, among others) and operational conditions affect the performance of wireless sensing units and the results from system identification algorithms executed by the sensors. Taking such a step will bring the field closer to the development of a self-interrogating wireless sensing system that provides civil engineers with accurate data and aids in subsequent data interpretation.

\section{DESIGN OF WIRELESS SENSING UNIT}

In this study, a wireless unit was developed to enable wireless data transmission from four channels of singleended arbitrary analog sensors, each with an output of 0 to 5 volts. Off-the-shelf microprocessor and radio development boards are employed, while a custom-designed ADC board is used to accommodate ADCs with 10-, 12- and 16-bit resolution, respectively. A low-pass filter was also designed for anti-aliasing purposes. The overall design is shown in Fig. 1.

The objective is to study noise contamination of the sensor data caused at different stages during the dataflow and further investigate how the noise will propagate into system identification result. A modular design was therefore adopted to facilitate the need to make a comparison of data quality based on different hardware options. As shown in Fig. 1, two types of analog sensors, ADCs with three different resolutions and radios with two different carrier frequencies are the variations considered in this study. An overview of each hardware components as well as software design is given as follows:

Analog Sensors Two commercially-available analog MEMS accelerometers, Analog Devices ADXL105EM-1 ${ }^{18}$ and Silicon Designs SD2012-005, ${ }^{19}$ with output signal ranges from 0 to $5 \mathrm{~V}$ are employed at different times with the wireless sensing unit. These specific accelerometers and others within their product families have been widely studied in the literature as part of structural monitoring studies centered on wireless sensing technologies.

ADC and LPF The first and the most critical stage when concerned with data quality is the analog-to-digital (ADC) conversion process. The ADC takes the analog signal from a sensor and converts it into a digital format which can be understood and processed by a microcontroller. In this study, ADCs with 10-, 12and 16-bit resolutions from the same vendor (Texas Instruments) are selected. It should be noted that all three ADCs have the same serial communication protocol for interfacing to the host microcontroller. Due to similar interfacing requirements, these ADCs were soldered onto a specially fabricated printed circuit board (PCB), creating a flexible system where different data acquisition boards can be readily connected to the microcontroller. The ADC channel pins were also brought out to a connector for easy connection to any analog sensor. To avoid aliasing of response data in the vicinity of the Nyquist frequency, a 4-pole Butterworth low-pass anti-aliasing filter (LPF) is designed from simple analog electrical components to have a cutoff frequency of $35 \mathrm{~Hz}$. It is recognized that a generically designed low-pass filter might distort measurements signals. Hence, the effect of the LPF on measurement data quality will be examined as part of this study.

Microcontroller A Motorola $68 \mathrm{HC} 11$ microcontroller mounted to a compact development board is selected as the computing element of the wireless sensor design. Also mounted to the development board is 32 KB SRAM, 32KB EEPROM, access pins for the SPI port and a built-in RS232 transceiver with a DB9 connector. The small amount of memory effectively limits the number of the data points that can be stored and subsequently sent by the radio. Based on this hardware limitation, it is decided to cap the size of time-history data streams at 12000 points in all the preliminary single channel tests described later. 

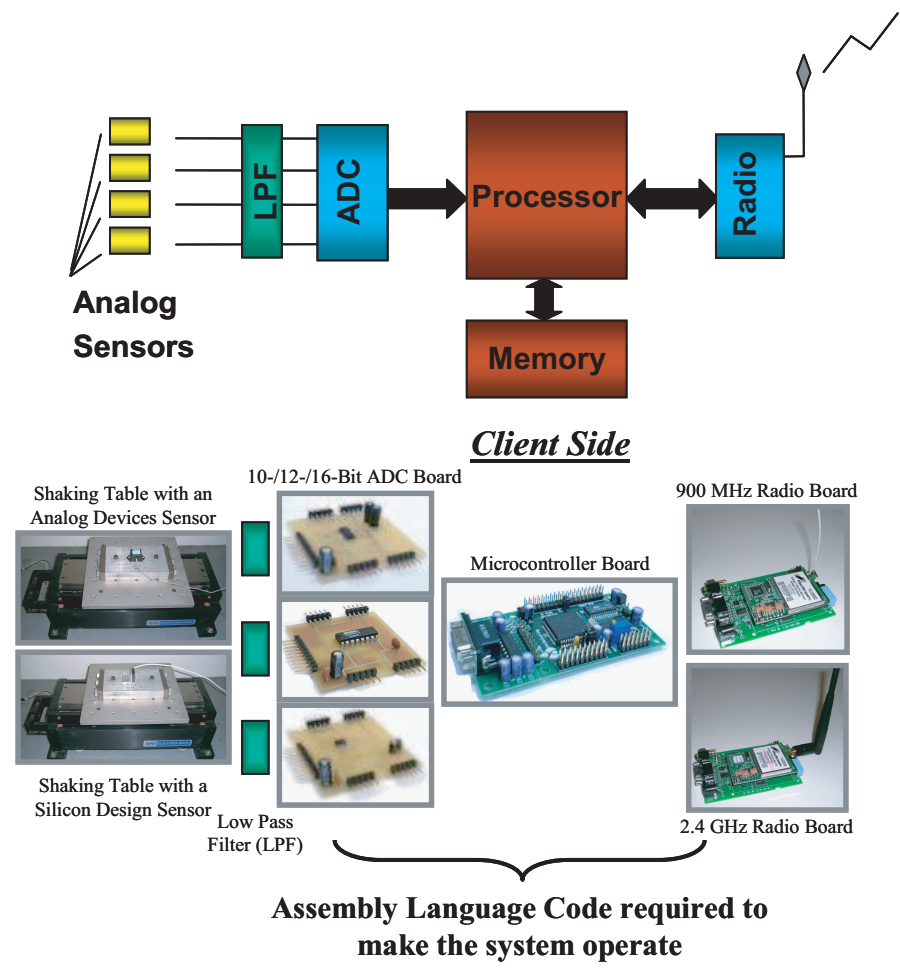

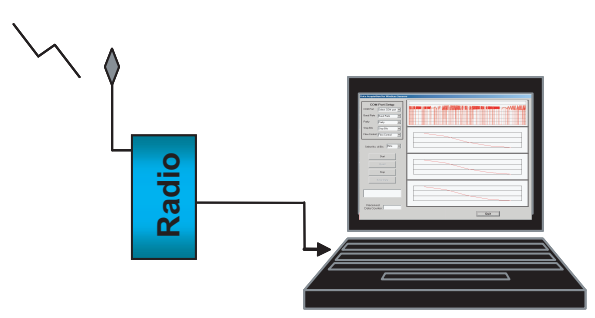

$\underline{\text { Server Side }}$

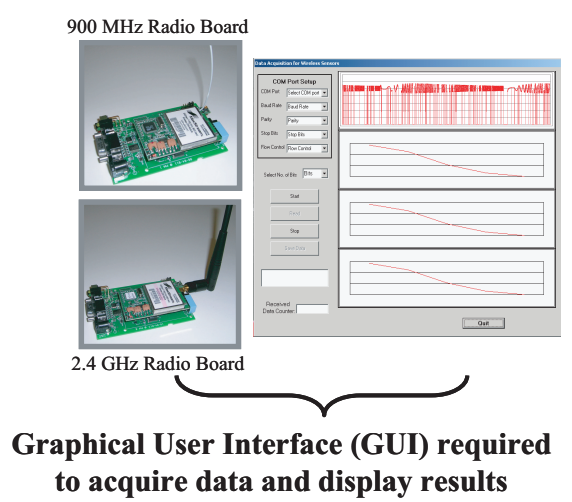

Figure 1. Various hardware options in this study. Shown here are the components of a wireless sensing unit developed to investigate the effect of critical hardware and operational condition to the data quality.

Radio For this study, original equipment manufacturer (OEM) radios are selected. Specifically, MaxStream radios that operate on the $900 \mathrm{MHz}$ and $2.4 \mathrm{GHz}$ frequency bands ${ }^{20}$ are chosen. These radios have been adopted in other studies. ${ }^{21,22}$ Both radios implement frequency hopping spreading spectrum (FHSS) as their primary modulation technique, thereby ensuring a high degree of reliability in the wireless channel. Since propagation and path losses have different characteristics at different frequencies, the choice of using two different radio bands provides the authors with the ability to thoroughly analyze the effect of the carrier frequency on the overall monitoring system performance. Also note that these two radios have different transmitting powers. Throughout this study, the $900 \mathrm{MHz}$ Maxstream radio was paired with a $1 / 4$ wave monopole whip antenna, while the $2.4 \mathrm{GHz}$ radio worked with a $1 / 2$ wave dipole antenna.

Software To investigate the data quality in a wireless setting, the assembly code and GUI were developed to capture loss of the data points by carrying out packetization to include various information including headers and packet ID.

\section{EXPERIMENTAL INVESTIGATIONS}

The experimental investigation is considered in two parts. In Part I, loopback tests were conducted using the MaxStream radios. These tests examine the transmitting range of the radios and characterize the transmitting environment. Based on the results of Part I, time histories of shaking table tests were collected at several locations that result in different degrees of packet loss. At each location, various combinations of hardware design options and operational conditions are studied.

All of the experiments are carried out inside and/or in the vicinity of the Fears Structural Engineering Laboratory (abbreviated as Fears Lab herein) at the University of Oklahoma. The laboratory is located in an open terrain landscape on the south campus of the university where the buildings are low-rise and sparse. The surrounding areas have a slightly higher elevation than the lab. Around some of the buildings in the testing area, there are a number of large trees. The tests reported herein are conducted spanning from late September to the 
beginning of March. This information is specified here since the foliage condition is expected to have an impact on wireless transmission especially in rural $\operatorname{areas}^{23}$; this investigation aims at weighting the contribution from this factor of signal attenuation. Vehicle traffic was light during the day time when the tests were conducted.

The building that houses the Fears Lab was constructed during the 1970's. On its west side, there is one high bay area with a strong floor, while an attached office area is on its east side. The high bay area is a steel structure of 24 feet high measured at the corners of the eastern and western edges. There is an 8-inch thick masonry unit wall around the high bay area from the ground up until the height of 8 feet. Above that, there is a 0.025 -inch thick steel sheet wall. An air-conditioned chamber is located inside the high bay area at its southeast corner where the shaking table is bolted down to the strong floor. The chamber has the same concrete masonry unit wall that is 8 -inch thick. The roof of the building is made of 2.5 -inch thick timber elements with a 10-inch insulation layer attached underneath. All these building materials will affect the transmitting range of the wireless connection as will be validated by the testing results.

This investigation offers a case study in which the focus is given to understanding how the data quality is affected by a low antenna elevation and when the radio transmits signals through building materials common to typical university structural engineering laboratories. Throughout this study, the transmitting and receiving radios were fixed at a height of six feet above the ground. A steel post was used to control the height of six feet at the client side, while the height of the server side was maintained as six feet by a student during communication with the transmitting wireless sensing unit. Since the wireless sensing unit must remain mobile, it was powered by a simple $9 \mathrm{~V}$ DC battery.

As mentioned above, the entire experimental study was conducted under either sunny or partially cloudy conditions on different days spanning half a year; the results presented here were collected within a winter month unless otherwise specified. All the tests were conducted during the daytime when there was little activity in the high-bay area. This was to minimize the influence from human activities. Except selecting testing time to accommodate weather and human activities, nothing else was done to specifically "design" a testing environment.

\subsection{Packet Delivery Performance}

To study the quality of the time history measurements transmitted and collected by using the designed wireless unit, the foremost step is to investigate the packet delivery performance during transmission. As the basic tool for this, packet loss rate means the fraction of the packets not successfully passing Cyclic Redundancy Check (CRC). ${ }^{13}$ The complimentary quantity of packet loss rate is packet reception rate.

In general, the causes for packet losses can be from both physical and protocol layers. ${ }^{13}$ In this selected testing environment, the possible factors for packet losses may be summarized as follows:

1. Beam divergence as the signal spreads while travelling away from it source, i.e., the influence from transmitting range.

2. Attenuation caused by building materials, i.e., the structural components (walls, roofs, columns and beams) of Fears laboratory.

3. Foliage attenuation, i.e., the trees surrounding the lab in the path of signal transmission.

4. Attenuation caused by other materials inside and surrounding the lab that can absorb electromagnetic energy. Examples are steel furniture and testing equipment.

5. Interference from vehicles.

6. Interference from other human activities.

Among the above list, some factors are relatively permanent (e.g., item 2) while some are changing (e.g., item 3) and may be considered as random (e.g., item 4). Some effects can be quantified (e.g., item 1 as studied in various theoretical and empirical equations for path $\operatorname{loss}^{14,15}$ ) while others are harder to quantify (e.g., items 5 and 6$)$. 
It is of great practical importance to differentiate these various factors influencing packet delivery performance, i.e., identify the controlling factors. Also it is essential to understand the nature of the packet loss process (i.e., its spatial and temporal characteristics) in addition to examining the packet loss pattern and its severity. These are the first steps to understand the issue of data loss and loss pattern associated with wireless sensing.

\subsection{Part I: Radio Loopback Tests}

Given the complexity related to predicting packet loss, experimental methods for characterizing the transmitting environment and determining the transmitting range are needed. Loopback tests were carried out at different server/laptop locations that corresponded to different transmission ranges while the wireless sensing unit was fixed at a location either inside or outside the Fears Lab; Some of the results are presented in Fig. 2.

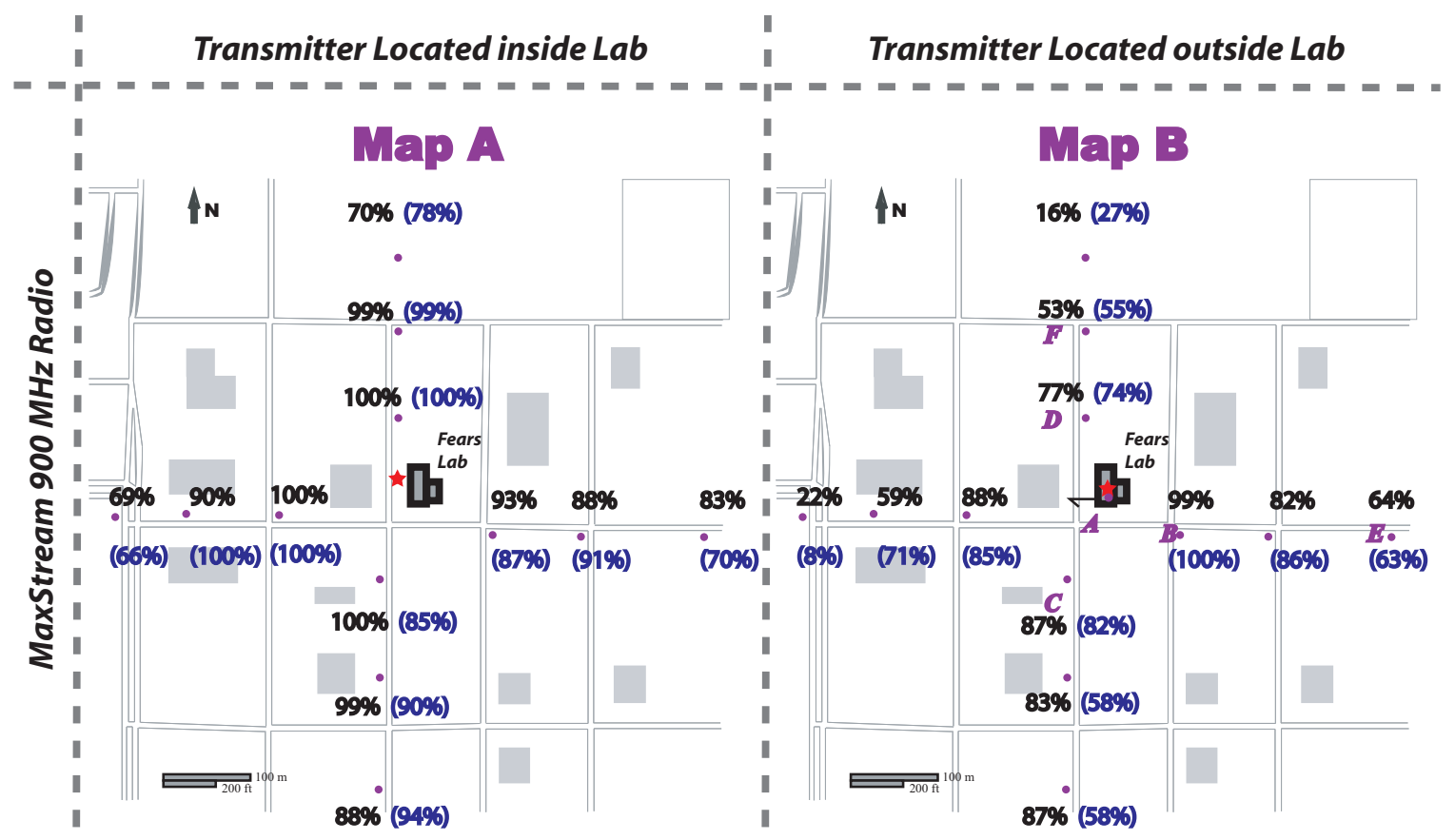

Figure 2. Results of loopback tests of MaxStream wireless OEM radios of $900 \mathrm{MHz}$. The client side (shown by a red star) was outdoor in Map A and indoor in Map B, respectively. In each map, multiple server/laptop locations where the loopback test was conducted are highlighted as purple dots with the average packet reception rate of four samples indicated at each location. In all tests, the heights of the client and server sides were fixed at six feet. The values in black were recorded in fall, while the values in blue and inside parentheses were recorded in winter.

Maps A and B in Fig. 2 have identical sets of server/laptop locations but different client locations. In each map, readings were taken along the four main directions. Multiple server/laptop locations where the loopback test was conducted are highlighted as purple dots. In Map B, six locations are specified from A to F, and they are the locations where the time histories of shaking table vibrations will be collected later on.

The loopback test was repeated four times at each server/laptop location to obtain an average value of packet reception rate. In each test, the server/laptop radio transmits 100 packets, each 32 bytes in size, to the client wireless sensing unit radio, which in turn returns all the received packets to the server radio. The software operating at the server end counts all the good packets that it has received and displays the appropriate result. The two sets of the results on each map were from the tests in two seasons. The first set, the values of which are in black were recorded in early fall. The second set, whose values in blue and inside parentheses were recorded in winter.

Maps A and B in Fig. 2 give a qualitative idea of the role played by transmitting range, effect of building materials and foliage attenuation. Before commenting on these results, it is important to bear in mind that every 
number is a mean value of 400 realizations of a random arrival process. The following comments are applicable to the statistical measures of such a random process rather than individual realizations. Qualitative conclusions about the building material attenuation can also be drawn by using Maps A and B as follows:

- Along each of the North, West and South directions, the packet reception is reduced distinctively when the transmitter is removed from the outside (see Map A) to the inside of the lab (see Map B). This can be explained by the addition of the walls of Fears lab that the signal has to penetrate in the later condition.

- The packet delivery performance of the East direction is affected adversely when the transmitter is moved from the outside (see Map A) to the inside (see Map B) in most locations. This is largely because of the increment in the number of walls that signal has to penetrate in the later condition.

- On Map A, transmission in the North and South directions is close to Line-of-Sight(LOS). Accordingly, the packet delivery performances along both directions are comparable. However, this comparison does not hold on Map B. The reduction in the packet delivery performance is less along the South than that along the North direction. This can be explained based on that fact that the transmission along the north direction on Map B is entirely blocked by the north and west steel sheet walls and perhaps the steel roof of Fears lab as well. The blockage from the building materials along the South direction, however, is only from the south steel sheet wall.

- The more severe reduction in the packet delivery performance along the West direction from Map A to B, as compared with that along the south direction can be similarly explained as above, adding more evidence for the strong effect of the attenuation from building materials.

- The difference between the results collected in fall and winter does not seem to be consistent, which may indicate that foliage attenuation, for this specific transmitting environment, is not a dominant factor in packet loss.

\subsection{Part II: Shaking Table Tests}

\subsubsection{Test setup}

In this study an electromagnetic shaking table was used to generate vibration signals. As shown previously in Fig. 1, the empty shaking table is instrumented with an accelerometer at the center of its top plate. An arbitrary wave form generator card $^{24}$ from National Instrument is used to generate a repetitive swept sine excitation to drive the empty shaking table. The card has a digital-to-analog resolution of 12 bits. The swept-sine starts at $0.10 \mathrm{~Hz}$ and ends at $20 \mathrm{~Hz}$ with a duration of 15 seconds. All of the shaking table data is steady-state response.

The data length is unified to be 12000 for all time histories collected as stated before. Such a data length permits the recording of one full run of the steady state response of the shaking table even at the $500 \mathrm{~Hz}$ sampling rate (see below). The cases of using the analog sensors with a tethered data acquisition system (i.e., "wired" cases) are employed as control experiments. In this test, sensor data is collected using a 16-bit NI DAQCard$6036 \mathrm{E}^{25}$ next to the location of the shaking table. When the wireless unit is used and when a packet is lost, the server inserts the artificial number of "0" in lieu of the true data point(s). Therefore a time history collected by the server can be readily converted into another one showing a blank spot for any data point lost in the wireless transmission so that the data loss can be easily calculated.

The purposes of the shaking table tests are two: 1) to examine the data loss and loss pattern of the transmitted time histories as influenced by the combined effect of transmitting range, building materials and other factors, and 2 ) to study how the hardware design options can affect the quality of the received data. Numerous combinations of hardware options and operational conditions are possible in the shaking table tests. Two main groups of combinations are made to fulfill the above two purposes, they are 1) different operational conditions - sampling rates of 500 and $100 \mathrm{~Hz}$, and six server/laptop locations (Locations A to F in Map B in Fig. 2), and 2) various hardware options including two analog sensors (ADXL105EM-1 and SD2012) and ADCs with resolutions of 10, 12 and 14 bits as shown in Fig. 1. Since different hardware configurations specified in the second group are not expected to affect data transmission, this results reported in this paper are focused on the effect of different server/laptop locations. 


\subsubsection{Overview and relation to loopback tests}

In stead of using transmitting range alone for characterizing packet delivery performance and data loss, this study seeks a justified application of the loopback test result. In addition to one indoor location (Location A), five outdoor locations (Locations B to F following the location IDs defined in Map B of Fig. 2) are selected from the loopback test results for collecting time histories of the shaking table tests based on the following considerations:

- The packet reception rates based on the loopback tests in both fall and winter represent a typical range of values, namely, from about $90 \%$ to $50 \%$ from Locations B to F. It would be interesting to see if the data reception results from the time histories would display the same trend.

- Locations B and D have about the same transmitting range, however, their loopback results are drastically different. Uncertain environmental factors may contribute to this difference, however building material attenuation seems to be the leading factor.

- Location $\mathrm{E}$ has the farthest transmitting range, however its loopback reception rate is not the lowest. This is another typical case to examine using the collected time histories.

Figure 3 presents the data reception rates calculated from the shaking table tests with the corresponding packet reception rates from the loopback tests overlaid as a comparison. In total, 54 data points from the shaking table tests are presented in the figure with the x-axis as the location ID and the y-axis, the data reception rates in percentage. The transmitting range is not chosen as a variable in this figure because transmitting range alone cannot entirely characterize the overall effect of signal attenuation and packet loss in this specific testing environment as discussed above.

It is important to note the differences between these two sets of receptions rates. The loopback test yields a packet reception rate from a loop-back mode following a fixed payload size scheme, while the oneway transmission-based shaking table test leads to a data reception rate by using a flexible payload size scheme. Strictly speaking, these two sets of rates are different qualities although they are closely related to each other. Furthermore, the loopback test in this study takes an average of only 400 packets. By contrast each time history contains 12000 data points, the statistical measures of which are quite representative. Nevertheless, the major connection between these two sets of results is that they are affected by the same transmitting range and similar environmental conditions.

It can be seen from Fig. 3 that the dispersion of the data points at Location $\mathrm{A}$ is the least given that it is an indoor location. At other outdoor locations, the reception rates vary in a range of about $15 \%$ to $35 \%$ at the same location. Different legends are used in Fig. 3 to indicate the realizations collected consecutively by using the same hardware configuration. Various hardware configurations in this study are not expected to lead to different packet delivery performance as stated previously, however, environmental factors including foliage, traffic flow, and human activities would affect packet delivery performance and data loss as different configurations were used on different days. Data successively collected on the same day have a dispersion of data loss of less than $25 \%$ in all outdoor cases, which is smaller than the range described above that includes tests done at the same location but on different days. This loosely demonstrates the influence from a wide range of unknown environmental impacts on the packet delivery performance and data loss.

With two exceptions (Locations B and F) out of the five outdoor locations, the data reception rates of the time histories are consistent with the packet reception rates from the loopback tests, which suggests the possibility of adopting the loopback as a fast prototyping means for characterizing a transmitting environment, although more realizations of the random process need to be collected and further theoretical and/or simulations are needs to justify this practical approach.

\subsubsection{Pattern of data loss}

Figure 4 demonstrates vividly the impact of packet loss to the time histories transmitted by the designed wireless sensing units. The reception rate tends to decrease from Locations A to F, i.e., the severity of packet loss increases from Locations A to F. It can be seen clearly that the lost data tend to be clustered in time. This phenomena imposes a challenge in numerical interpolation to recover the missing data points. 


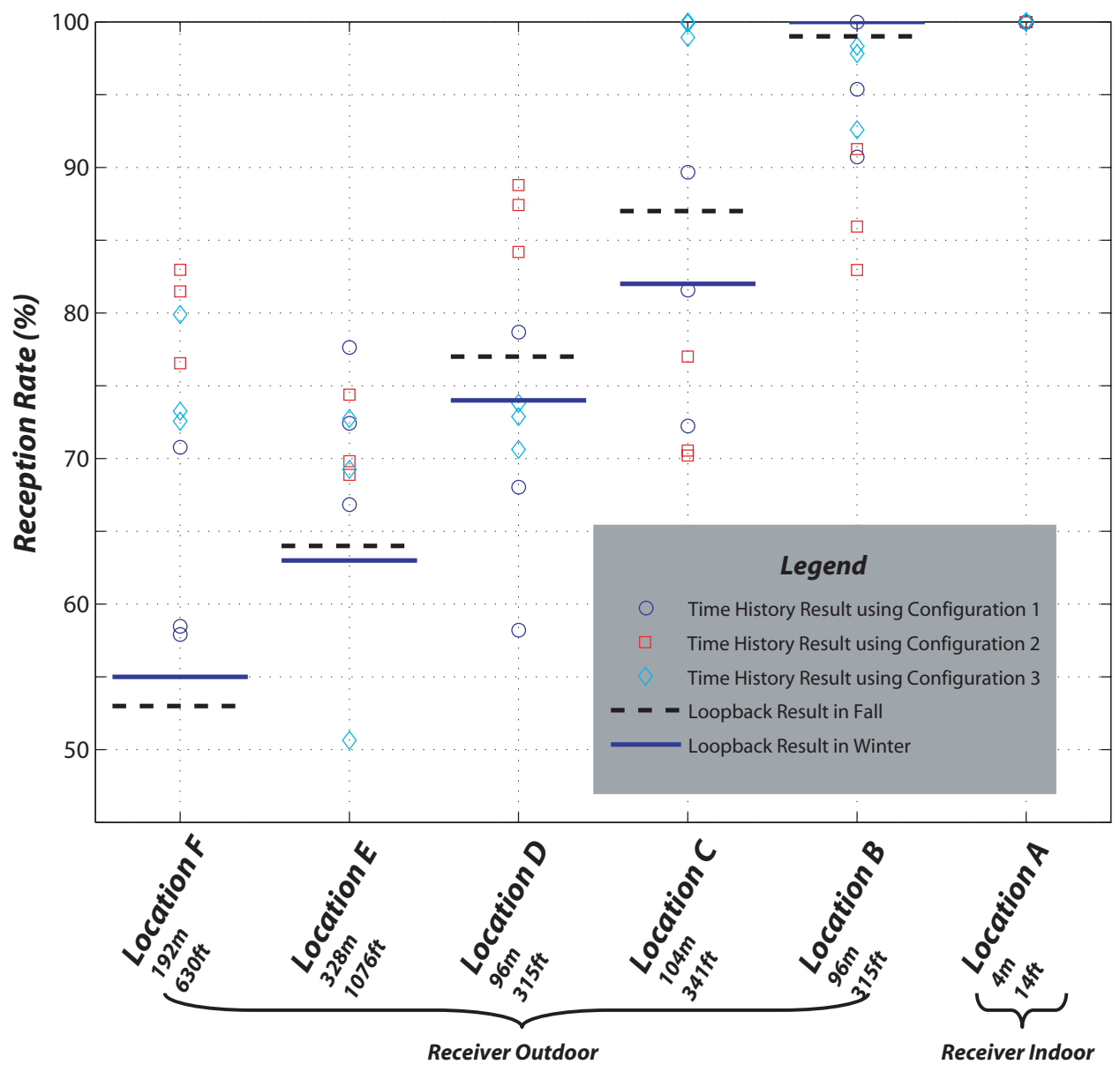

Figure 3. Reception rates calculated from transmitted shaking table time histories and those based on loopback tests. The values of transmitting range for each locations are indicated in the figure. The difference in these hardware configurations 1, 2 and 3 would not be expected to impact packet delivery performance. Instead, they reflect the influence from the environmental factors such as foliage, traffic flow, and human activities.

\section{DISCUSSIONS AND CONCLUSIONS}

The above preliminary experimental results have demonstrated the challenges in designing a wireless sensing unit. In particular, they clearly show how critical the reliability of the wireless communication channel is when transmitting data over large spatial distances, as would be common in structural health monitoring. Packet losses caused by building materials and transmitting ranges must be reduced if data is to be used for detecting structural damage. Possible solutions include, but are not limited to, 1) raising the height of the antenna, however this needs further scrutiny, especially for the nodes in a decentralized wireless sensor network, and 2) increasing the complexity of the software in the design of the wireless unit to re-transmit any lost data sets (as is common in sophisticated network protocols like TCP/IP). Nevertheless, this second solution might not be suitable for a real-time health monitoring system and might not to be cost effective for applications to civil infrastructures. The results in this study show clearly the necessity of developing smart wireless sensing techniques. If data were collected and processed onboard using an embedded system, the result to be transmitted would no longer necessarily be a sensitive raw time history, but rather some simplified indicators that alert building owners to the status of the monitored structure. Thus the adverse effect of packet loss can be reduced through local data interrogation.

Unlike a cable-based monitoring system, the issue of missing data has to be addressed in a wireless sensor network. Numerical interpolation would be an effective means to restore the missing data points when the data 

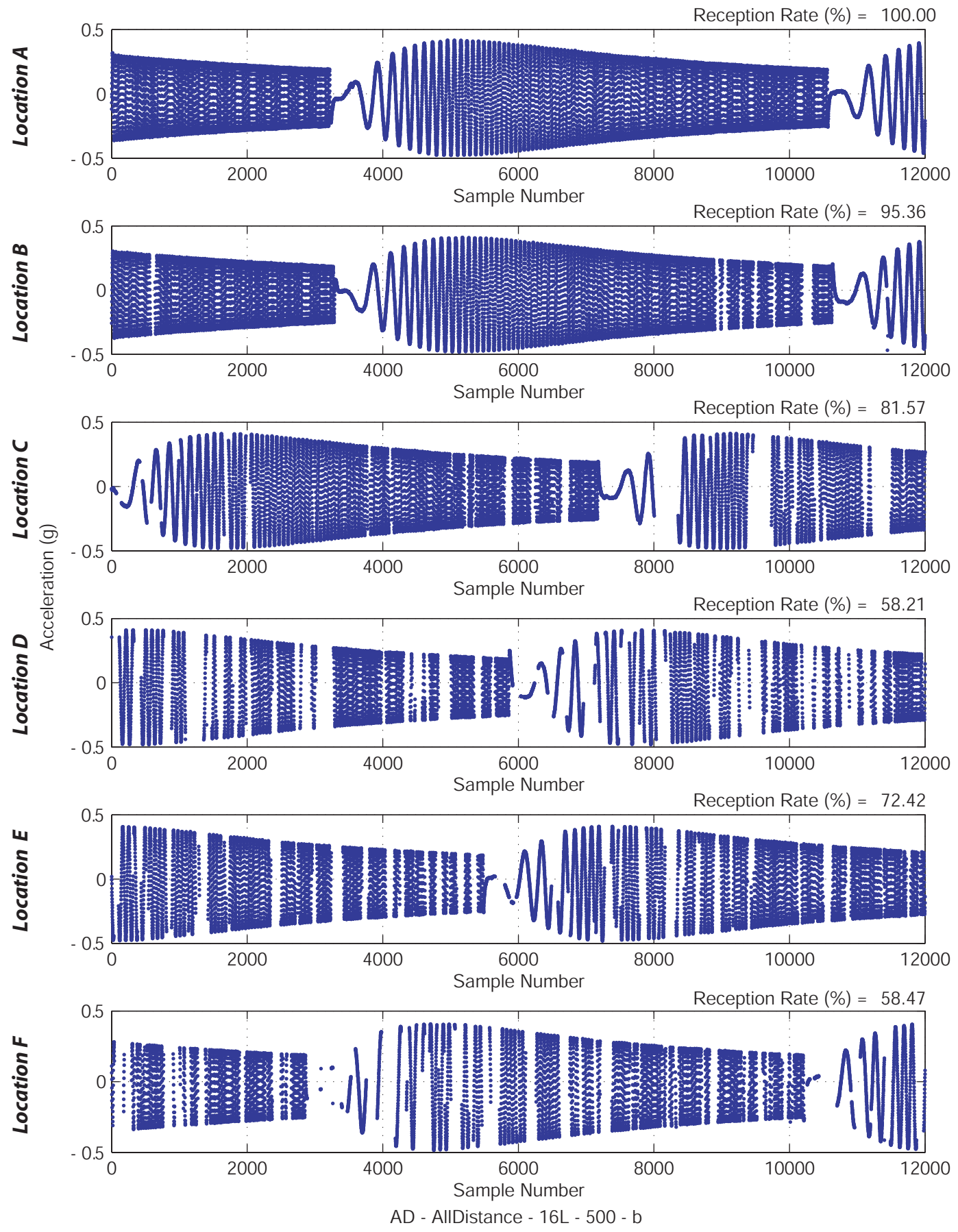

Figure 4. Time histories of sample set b using ADXL105EM-1 and 16-bit ADC with LPF from Locations A to F with a decreasing anticipated reception rate from $100 \%$ to $50 \%$. Note that the reception rates calculated from each measured time histories are indicated on the top right corner of each panel. 
loss is sufficiently low and the signal pattern is fairly simple; however, a numerical interpolation becomes an extrapolation when the missing points are clustered. In that case, the trend line would be hard to capture especially for a nonstationary process and/or nonlinear response. Statistical approaches should also be exercised to fuse data ${ }^{26}$ especially by condensing "redundant" sets of data to achieve a data set with a reduced loss rate to enable further numerical interpolation. What has been demonstrated in this study is the case of applying a deterministic excitation to a deterministic system. How to handle more complicated situations remains a challenge that the authors will address in a further study.

\section{ACKNOWLEDGMENTS}

This study is supported by the National Science Foundation (SGER CMS-0332350) under Program Officer Dr. Steven L. McCabe. The Junior Faculty Research Program awarded to the first author by Dr. T.H. Lee Williams, the Vice President for Research at the University of Oklahoma (OU) is greatly appreciated. The support from the university program at Analog Devices, Inc. in offering their MEMS accelerometers is gratefully acknowledged. In addition, the assistance offered by Mr. Mike F. Schmitz throughout the laboratory testing is deeply appreciated. The assistance offered by Miss Anh P. Nguyen at OU in conducting the transmission range tests is acknowledged. Two REU students from Industrial Engineering, Miss Elizabeth Henry from the SUNY Buffalo and Miss Melissa Lindner from the University of Oklahoma are thanked for their participation and assistance during the preliminary test in summer 2004 .

\section{REFERENCES}

1. E. Straser and A. Kiremidjian, "A modular, wireless damage monitoring system for structures, report no. 128," tech. rep., John A. Blume Earthquake Engineering Center, Department of Civil and Environmental Engineering, Stanford University, 1998.

2. J. Lynch, A. Partridge, K. Law, T. Kenny, A. Kiremidjian, and E. Carryer, "Design of a piezoresistive mems-based accelerometer for integration with a wireless sensing unit for structural monitoring," Journal of Aerospace Engineering, ASCE 16(3), pp. 108-114, 2003.

3. J. Lynch, A. Sundararajan, K. Law, A. Kiremidjian, E. Carryer, H. Sohn, and C. Farrar, "Field validation of a wireless structural monitoring system on the alamosa canyon bridge," (San Diego, CA), March 2-6 2003. SPIE 10th Annual International Symposium on Smart Structures and Materials.

4. J. Lynch, A. Sundararajan, K. Law, A. Kiremidjian, T. Kenny, and E. Carryer, "Embedment of structural monitoring algorithms in a wireless sensing unit," Structural Engineering and Mechanics, Techno Press 15(3), pp. 285-297, 2003.

5. N. Kurata, B. Spencer, M. Ruiz-Sandoval, Y. Miyamoto, and Y. Sako, "A study on building risk monitoring using wireless sensor network mica mote," in Structural Health Monitoring and Intelligent Infrastructure, pp. 353-357, (Tokyo, Japan), 13-15 November 2003. Proceedings of the First International Conference on Structural Health Monitoring and Intelligent Infrastructure, edited by Z.S. Wu and M. Abe, Swets \& Zeitlinger B.V., Lisse, The Netherlands.

6. M. Ruiz-Sandoval, B. Spencer, and N. Kurata, "Development of a high sensitivity accelerometer for the mica platform," pp. 1027-1034, (Stanford, CA), September 15-17 2003. Proceedings of the 4th International Workshop on Structural Health Monitoring.

7. B. Spencer, "Some real-world applications of wireles sensor nodes," in Smart Structures and Materials 2004: Sensors and Smart Structures Technologies for Civil, Mechanical and Aerospace Systems, pp. 344-355, (San Diego, CA), March 2004. Proceedings of SPIE.

8. L. Donoho, "Wireless develops extrasensory vision of future," Journal of New England Technology, 2003.

9. S. Seidel and T. Rapport, "914 mhz path loss prediction model for indoor wireless communication in multifloored buildings," IEEE Transactions on Antennas and Propagation 40, pp. 207-217, February 1992.

10. H. Wang and N. Moayeri, "Finite state markov channel - a useful model for radio communication channels," IEEE Transactions on Vehicular Technology 44, pp. 163-171, February 1995.

11. K. Lee and S. Chanson, "Packet loss probability for real-time wireless communications," IEEE Transactions on Vehicular Technology 51, pp. 1569 - 1575, November 2002. 
12. C. Tang and P. McKinley, "Modeling multicast packet losses in wireless lans," in Proceedings of the 6th ACM international workshop on Modeling analysis and simulation of wireless and mobile systems, pp. 130 - 133, ACM Press, (San Diego, CA), 2003.

13. J. Zhao and R. Govindan, "Understanding packet delivery performance in dense wireless sensor networks," in Proceedings of the 1st international conference on Embedded networked sensor systems, pp. 1-13, ACM Press, (Los Angles, CA), November 2003.

14. P. Shankar, Introduction to Wireless Systems, John Wiley, 2002.

15. J. Mark and W. Zhuang, Wireless Communication and Networking, Prentice Hall, 2003.

16. F. Casciati, L. Faraveli, and F. Borghetti, "Wireless links between sensor-device control stations in long span bridges," in Smart Structures and Materials 2003: Smart Systems and Nondestructive Evaluation for Civil Infrastructures, pp. 1-7, (San Diego, CA), March 2003. Proceedings of SPIE Vol. 5057(2003), edited by S.-C. Liu.

17. A. Smyth and R. Betti, eds., Proceedings of the Fourth International Workshop on Structural Control, International Association on Structural Control (IASC), DesTech Press, 2004.

18. Analog, Devices Inc., ADXL105/ADXL150/ADXL250/ADXL190 Evaluation Modules, 2000.

19. Silicon, Designs Inc., Model 2012 Analog Accelerometer Module, 2000.

20. MaxStream, Inc., XStream-DEV Wireless Development Kits 900 MHz $\&$ 2.4 GHz, 1998-2003.

21. F. Casciati, S. Casciati, L. Faraveli, and R. Rossi, "Hybrid wireless sensor network," in Smart Structures and Materials 2004: Sensors and Smart Structures Technologies for Civil, Mechanical and Aerospace Systems, pp. 308-313, (San Diego, CA), March 2004. Proceedings of SPIE Vol. 5391(2004), edited by S.-C. Liu.

22. J. Lynch, A. Sundararajan, K. Law, H. Sohn, and C. Farrar, "Design of a wireless active sensing unit for structural health monitoring," in Smart Structures and Materials 2004: Sensors and Smart Structures Technologies for Civil, Mechanical and Aerospace Systems, pp. 157-168, (San Diego, CA), March 14-18 2004. Proceedings of SPIE Vol. 5391(2004), edited by S.-C. Liu.

23. E. Tameh and A. Nix, "The use of measurement data to analyse the performance of rooftop diffraction and foliage loss algorithms in a 3-d integrated urban/rural propagation model," in 48th IEEE Vehicular Technology Conference, pp. 303-307, 1998.

24. National Instruments, 40MS/s Arbitrary Waveform Generator.

25. National Instruments, Portable E Series Multifunction DAQ.

26. L. Klein, Sensor and Data Fusion, A Tool for Information Assessment and Decision Making, SPIE Press, 2004. 